\title{
Gesundheitsfonds
}

\section{Kassen erhalten Milliardenspritze aus der Reserve}

Die gesetzlichen Krankenkassen erhalten im nächsten Jahr 1,5 Milliarden Euro aus den Reserven des Gesundheitsfonds. Für diese einmalige Finanzspritze hat das Bundskabinett Mitte im August grünes Licht gegeben. Nach Regierungsangaben soll mit einer Milliarde Euro die vorübergehende Mehrbelastung der Kassen im Zusammenhang mit der gesundheitlichen Versorgung von Flüchtlingen finanziert werden. Weitere 500 Millionen Euro fließen demnach in den Aufbau der Telematikinfrastruktur im Gesundheitswesen. Zudem möchte die Regierung durch diesen Beschluss eine Schwankung der Zusatzbeiträge vermeiden. Hintergrund dafür dürften die anstehenden Bundestagswahlen 2017 sein.

Erst Mitte Juli hatte die Vorsitzende des Spitzenverbandes der Gesetzlichen Krankenkassen (GKV-Spitzenverband), Doris Pfeiffer, für das nächste Jahr einen Anstieg des Zusastzbeitrags um 0,3 Prozentpunkte auf 1,4 Prozent prognostiziert. In drei
Jahren werde die zusätzliche Belastung der gesetzlich Versicherten voraussichtlich sogar bei 1,8 Prozent liegen. Für Pfeiffer hat der Gesetzgeber selbst „durch seine kostspieligen Reformen der letzten Jahre wesentlich dafür gesorgt, dass die Zusatzbeiträge trotz sehr guter Einnahmen weiter steigen müssen." Im Gegensatz zum Bundeskabinett sieht die GKV-Spitzenverbandschefin keine Mehrbelastung der Kassen durch die Versorgung von Flüchtlingen.

Aktuell verfügt der Gesundheitsfonds über eine Reserve von rund zehn Milliarden Euro. Der Fonds setzt sich aus den Beitragseinnahmen der Kassen und Zuschüssen aus dem Bundeshaushalt zusammen. Nach bestimmten Kriterien erhalten die Kassen monatliche Zahlungen aus dem Fonds. Genügen die Zuwendungen nicht, können die Kassen Zusatzbeiträge von den Versicherten erheben. Der Bundesrat muss der beschlossenen Finanzspritze nicht mehr zustimmen.

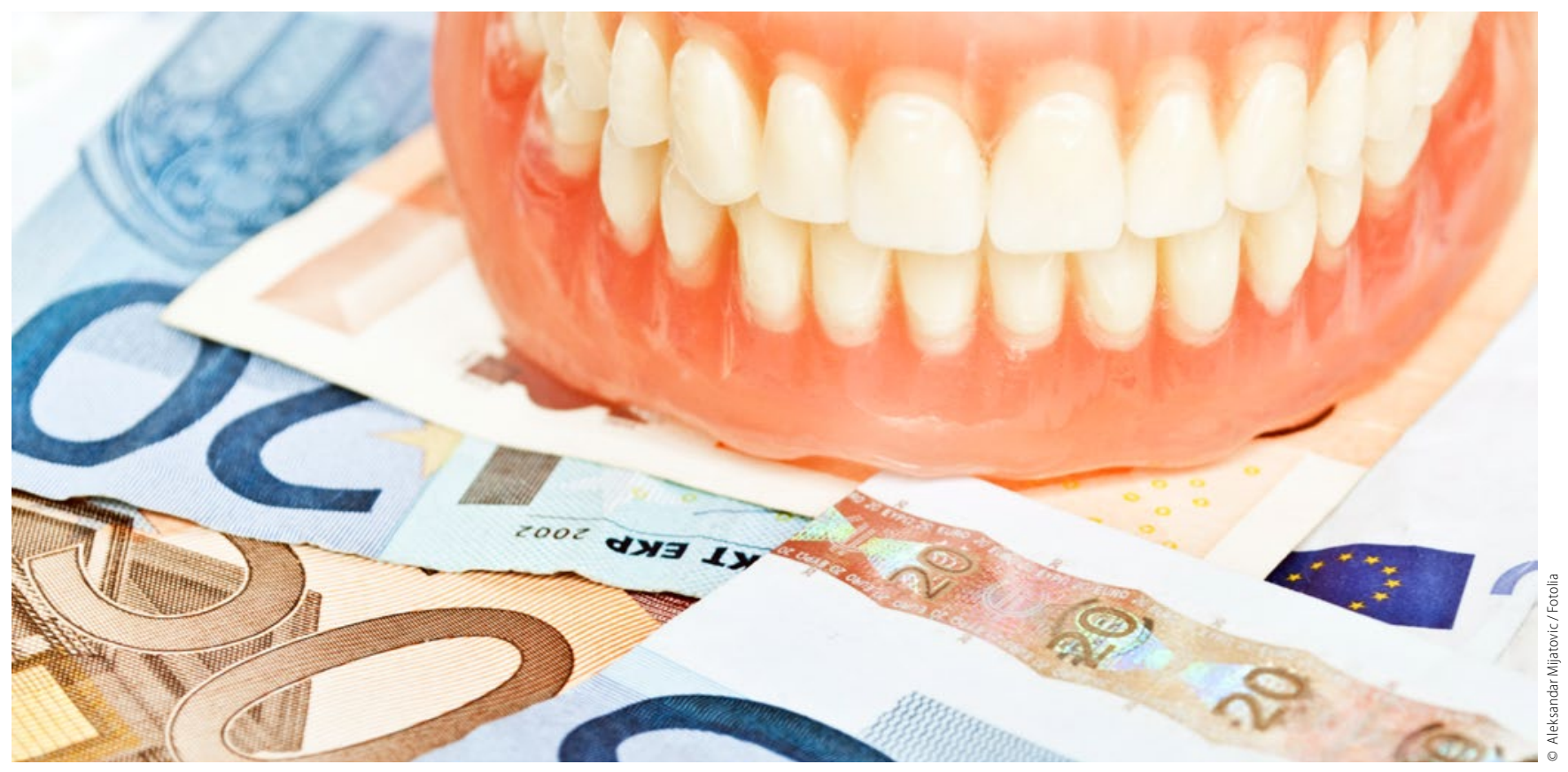

\section{Umfrage zum Zahnersatz}

\section{Dritte Zähne in jedem zweiten Mund}

Bereits heute ist Zahnersatz schon für mehr als jeden zweiten Bundesbürger (52 Prozent) Realität. Das ist das zentrale Ergebnis einer aktuellen Umfrage des Meinungsforschungsinstituts INSA im Auftrag des Verbandes der Privaten Krankenversicherung (PKV) unter 2000 Deutschen. Dabei gibt es zwischen den Geschlechtern lediglich geringe Unterschiede. Laut Umfrage benötigen Frauen Zahnersatz geringfügig häufiger als Männer. Die Mehrheit der Befragten, rund 54 Prozent, die aktuell noch keine Kronen, Brücken, Implantate oder Prothesen haben, geht der Umfrage zufolge davon aus, später Zahnersatz in Anspruch nehmen zu müssen.
Die Ergebnisse spiegeln sich auch in der Anzahl der abgeschlossenen privaten Zahnzusatzversicherungen wider: Bereits 2015 hatte der PKV-Verband einen Rekordwert vermelden können. Zwischen 2005 und 2015 hat sich nach PKV-Angaben die Zahl der Zahnzusatz-Policen auf rund 15 Millionen fast verdoppelt und nimmt damit einen Großteil aller privaten Zusatzversicherungen ein. Der PKV-Verband begründet den Anstieg vor allem mit Leistungskürzungen der gesetzlichen Krankenversicherung (GKV) bei der Übernahme der Kosten für Zahnersatz und Zahnbehandlungen. 